\title{
Untersuchungen über die chemische Zusammensetzung des Zellsaftes der Diatomee Coscinodiscus wailesii (Bacillariophyceae, Centrales)
}

\author{
HANSWERNER KeSSELER \\ Biologische Anstalt Helgoland, Litoralstation, List/Sylt
}

\begin{abstract}
Investigations on the chemical composition of the cell sap in the diatom Coscinodiscus roailesii (Bacillariophyceae, Centrales). A sample of cells of the plankton diatom Coscinodiscus wailesii was cultivated at $15^{\circ} \mathrm{C}$ under 14 hours illumination per day by fluorescent lamps (ca. 1000 lux). The aerated culture medium consisted of Erdschreiber solution of about $30 \%$ salinity enriched (after LEwIN 1965) with B $\left(5 \cdot 10^{-5} \mathrm{~m}\right.$ ) and provided with infusorial earth $(1 \mathrm{~g} / \mathrm{l})$ as a source of $\mathrm{Si}$ and trace elements. When the cell concentration reached about 100 per $\mathrm{ml}$ the diatoms were sifted off by a piece of plankton cloth (mesh width $200 \mu$ ) and rinsed on the sieve by artificial Li-sea-water. After this treatment most of the Li-solution still adhering was removed by filter paper; the remaining rest imbibed in the free accessible room of the cytoplasm as well as in the cell walls and in interstitial rooms between cells was determined by flame photometry as apparent free space (AFS). All results were obtained from a single sample only; they have therefore to be regarded as preliminary. Comparing these results with the information revealed by former investigations on the cell sap of other marine algae (Kesseler 1964, 1966) no uncommon facts could be established: Potassium and chloride proved to be the main intracellular ions, while sodium was restricted to about 0.25 times its medium concentration; $\mathrm{NH}_{4}{ }^{\circ}$ was not detectable. $\mathrm{Mg}{ }^{*}, \mathrm{Ca}{ }^{*}$, and $\mathrm{H}_{2} \mathrm{PO}_{4}{ }^{\prime}$ were present in relatively small amounts, while the sulfate concentration was rather high, and thus obviously compensating for the deficit in chloride ions. Similar relations, but more pronounced, were found in Desmarestia viridis. Acidity of cell sap, determined by means of Merck indicator paper, was $p_{H} 5$. Considering the dilution of the sample by the artificial Li-sea water, and the buffering effect of $\mathrm{H}_{2} \mathrm{PO}_{4}$ ions (probably from the cytoplasm), the $\mathrm{p}_{\mathrm{H}}$ of the original sap might have been about 4 . The specific gravity of artificial sap (prepared in agreement with the analytical results and determined arcometrically) was $1,0287 \mathrm{~g} / \mathrm{cm}^{3}$ at $20^{\circ} \mathrm{C}$, while the density of the culture medium, at the same temperature, was $1,0243 \mathrm{~g} / \mathrm{cm}^{3}$. The chemical properties of the cell sap of Coscinodiscus wailesii provide in no way suitable means of buoyancy, as could be demonstrated for the cell sap of Noctiluca (Kesseler 1966). This finding contradicts results by the Russian investigators BexLemishev, Petrixova \& Semina (1961) employing ultramicrochemical methods on samples of cell sap from single cells of the rather closely related giant species Etbmodiscus rex.
\end{abstract}

\section{EINLEITUNG}

Bereits an anderer Stelle wurde ein neues Verfahren ausführlich beschrieben, mit dessen Hilfe es gelang, auch von kleinzelligen Meeresorganismen genügend Zellsaft 
zu gewinnen, um darin quantitative Bestimmungen der osmotisch wichtigsten anorganischen Komponenten mit herkömmlichen chemischen Nachweismethoden durchführen zu können (Kesseler 1964). Durch Anwendung dieser Methodik gelang es unter anderem, die Frage nach der Ursache des Auftriebs der Zellen von Noctiluca miliaris, die sich an ruhigen, warmen Tagen in großen Mengen an der Meeresoberfläche ansammeln, befriedigend zu lösen (KESSELER 1966). Dieser Erfolg ermutigte zu dem Versuch, auch das Problem der Schwimmfähigkeit anderer Planktonorganismen, insbesondere yon Diatomeen, die trotz ihrer relativ schweren Kieselschalen bei ruhiger Wetterlage nur in der oberen euphotischen Schicht in großer Zahl anzutreffen sind, auf dem Umwege über eine Analyse ihres Zellsaftes einer Lösung näherzubringen; gibt es doch über diese interessante und oft diskutierte Frage außer theoretischen Abhandlungen, die sich auf Beobachtungen über die Sinkgeschwindigkeit dieser Organismen stützen (SMAYDA \& BOLEYN 1965, dort weitere Literatur), bisher lediglich eine Veröffentlichung, in welcher Analysenergebnisse über die chemische Zusammensetzung des Zellsaftes von Ethmodiscus rex mitgeteilt worden sind (Bekremishev, Petrikova \& Semina 1961). Diese, durch Anwendung ultramikrochemischer Nachweisverfahren gewonnenen Ergebnisse, auf die im folgenden noch näher einzugehen sein wird, stehen allerdings in krassem Widerspruch zu meinen Resultaten. Unter diesen Umständen erscheint es gerechtfertigt, die bisher erst an nur einer Zellsaftprobe erarbeiteten Analysendaten als vorläufige Ergebnisse zu veröffentlichen. Ein abschließendes Urteil über die noch offenen Fragen muß weiteren Untersuchungen vorbehalten bleiben, die hoffentlich auch dazu beitragen werden, die zur Zeit unüberbrückbaren Widersprüche zwischen den Ergebnissen der russischen Autoren und den eigenen Befunden zu Iösen.

Nach Uberwindung der Schwierigkeiten, die nach der Verlegung des botanischphysiologischen Laboratoriums von Helgoland nach List auf Sylt infolge unzulänglicher Arbeitsbedingungen zunächst auftraten, hoffen wir, auch die Untersuchungen an Planktondiatomeen bald wiederaufnehmen zu können.

\section{MATERIAL UND METHODIK}

Als Versuchsobjekt diente die sehr großzellige zentrische Planktondiatomee Coscinodiscus wailesii $(\varnothing \mathrm{ca} .350 \mu$ ). Durch die freundliche Vermittlung von Herrn Dr. R. Eppley (La Jolla) erhielt ich einen Ansatz dieses für meine Zwecke günstigen Materials aus den Kulturen von Herrn Dr. R. Holmes (La Jolla). Diese Probe wurde in einem auf $15^{\circ} \mathrm{C}$ temperierten Konstanzraum des Helgoländer Instituts bei täglich 14stündiger Beleuchtung durch Leuchtstofflampen (ca. $1000 \mathrm{Lux}$ ) und ständiger Belüftung mit Unterstützung von Herrn Dr. G. Drebes in Kultur genommen. Allen drei Herren sei an dieser Stelle für ihr freundliches Entgegenkommen recht herzlich gedankt.

Als Kulturgefäß diente eine Weithalsflasche aus Jenaer Glas von 101 Fassungsvermögen, die mit Erdschreiberlösung [Seewasser + Erdabkochung $+\mathrm{KNO}_{3}\left(10^{-3} \mathrm{~m}\right)$ $\left.+\mathrm{Na}_{2} \mathrm{HPO}_{4} \cdot 12 \mathrm{H}_{2} \mathrm{O}\left(5,5 \cdot 10^{-5} \mathrm{~m}\right)\right]$ von etwa $30 \%$ Salzgehalt gefüllt wurde. Gemäß den Befunden LEwrss (1965) wurde dem Kulturmedium noch $\mathrm{H}_{3} \mathrm{BO}_{3}$ $\left(5 \cdot 10^{-5} \mathrm{~m}\right)$ zugesetzt. Als $\mathrm{SiO}_{2}$-Quelle und Reservoir für weitere Spurenelemente diente gereinigte Kieselerde (MERCK) die in einer Konzentration von $1 \mathrm{~g} / 1$ zugegeben 
wurde. In dieser Kulturlösung vermehrten sich die Diatomeen recht gut, so daß nach etwa drei Wochen genügend Material für eine erste Bestimmung der Zellsaftzusammensetzung vorhanden war. Mit zunehmender Zellkonzentration nahmen die Diatomeen allerdings eine dunklere Färbung an, zeigten im übrigen jedoch außer gelegentlichen Spontanplasmolysen keine abnormen Veränderungen.

Zur Gewinnung des Zellsaftes wurde der Kulturansatz mit Hilfe eines PVCSchlauches über ein Sieb aus Planktongaze (Maschenweite $200 \mu$ ) vorsichtig abgehebert. Überschüssiges Tropf- und Haftwasser wurde unter Zuhilfenahme eines Zellstoffbausches von der Siebunterseite abgetupft, und das Sieb sodann für kurze Zeit (ca. 10 Sekunden) in künstliches Li-Seewasser vereinfachter Zusammensetzung (vgl. Kesseler 1964) getaucht. Durch Vergleich der mikrokryoskopisch (Kesseler 1958) bestimmten Gefrierpunktserniedrigungen des Kulturmediums und der Li-Stammlösung und entsprechendes Verdünnen der letzteren mit destilliertem Wasser wurde Isotonie beider Medien garantiert; damit konnten osmotische Wasserverschiebungen als mögliche Fehlerquelle ausgeschaltet werden. Die Reste des anhaftenden beziehungsweise in dem interstitiellen Raum zwischen den Zellen befindlichen Kulturmediums wurden durch mehrmaliges Übergießen mit insgesamt $100 \mathrm{ml}$ Spüllösung entfernt, die überschüssige Li-Lösung, wie oben beschrieben, abgetupf, und die Diatomeen samt Planktongaze sodann in ein Zentrifugenglas unter Paraffinöl gebracht. Nach Abtötung des Materials durch Tiefgefrieren bei $-20^{\circ} \mathrm{C}$ wurde die Probe in einen Kühlschrank gebracht und dort bei einer Temperatur von ca. $+2^{\circ} \mathrm{C}$ langsam wieder aufgetaut. Die Gewinnung des Saftes erfolgte durch 30 Minuten langes Zentrifugieren in der Ultrazentrifuge (BECKMAN "Spinco L") bei 8000 U.p.M. (ca. $9000 \mathrm{~g}_{\mathrm{max}}$ ) unter Verwendung eines Schwingbecherrotors (SW 25.1). Auf diese Weise wurde etwas mehr als $0,5 \mathrm{ml}$ Saft gewonnen, der allerdings zu ca. 50\% durch die Li-Spüllösung verdünnt war. Diese Menge entspricht dem Vakuoleninhalt von etwa $10^{5}$ Zellen beziehungsweise einer Zellkonzentration von etwa 100 Zellen pro ml Kulturlösung. Der gewonnene Saft wurde schließlich nochmals bei 25000 U.p.M. (ca. $90000 \mathrm{~g}_{\max }$ ) nachzentrifugiert. Nach dieser Behandlung war er völlig klar und nur von schwach gelblich-brauner Färbung. Zur Bestimmung von $\mathrm{Cl}^{\prime}, \mathrm{SO}_{4}{ }^{\prime \prime}, \mathrm{Ca}$ " und $\mathrm{Mg}$ " mit Hilfe der bereits an anderer Stelle mitgeteilten Nachweisreaktionen (KESSELER 1964) wurde er im Verhältnis 1;50 mit destilliertem Wasser verdünnt. $\mathrm{Li}^{*}, \mathrm{Na}$ und $\mathrm{K}$ wurden bei einer Gesamtverdünnung von 1:250 flammenphotometrisch ermittelt, während sich zur Bestimmung von $\mathrm{NH}_{4}$ (nach Nessler) beziehungsweise $\mathrm{P}$ Verdünnungen von $1: 500$ respektive $1: 2500$ als optimal erwiesen.

\section{ERGEBNISSE UND DISKUSSION}

Die Ergebnisse der chemischen Analysen, die unter Berücksichtigung des aus der Lithiumbestimmung ermittelten AFS (extravakuolarer Lösungsraum des Protoplasmas + Imbibitionsraum der Zellwände + interstitieller Raum + Haftwasservolum) auf ursprüngliche Zellsaftkonzentration umgerechnet wurden, sind in Tabelle $1 \mathrm{zu}$ sammengestellt. 
Tabelle 1

Zusammensetzung des Zellsaftes von Coscinodiscus wailesii aus einem Kulturmedium von $32,7 \%$ Salzgehalt

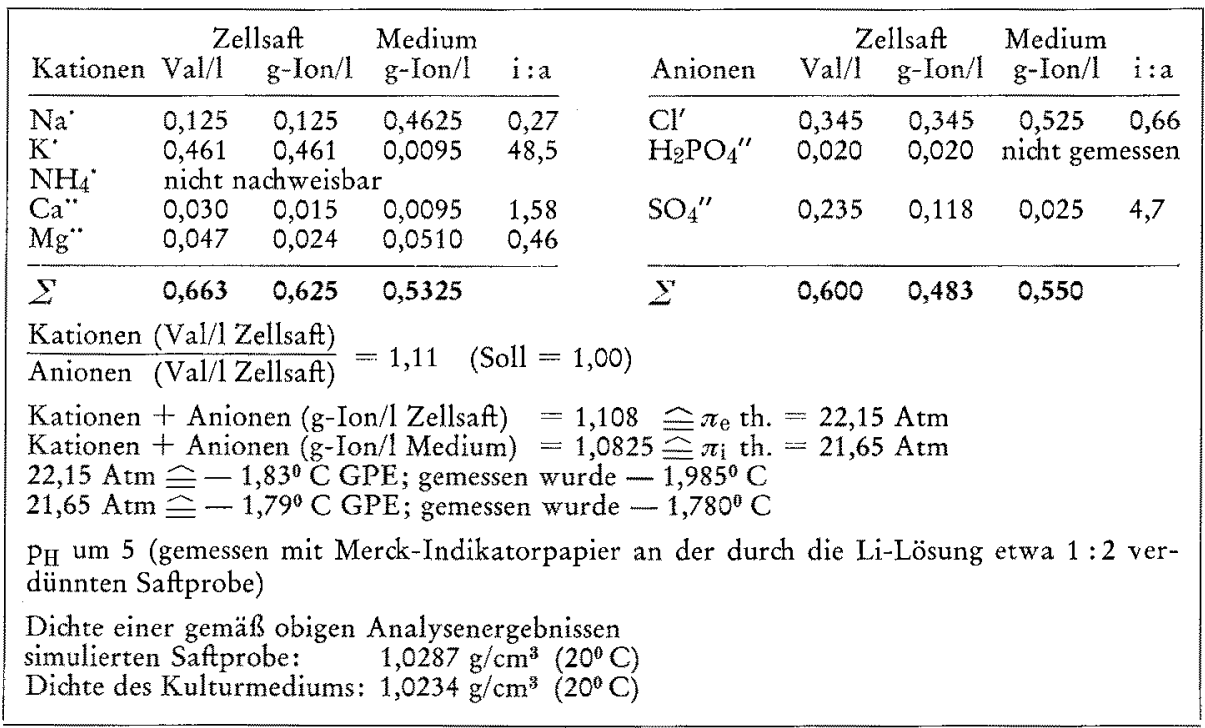

In dieser Tabelle sind die errechneten Zellsaftkonzentrationen der verschiedenen Ionenarten einmal in Aquivalent pro Liter (Val/1) zur Veranschaulichung der stöchiometrischen Verhältnisse, zum anderen in Grammion pro Liter (g-Ion/l) zwecks leichterer Uberschaubarkeit ihrer osmotischen Wirkungen aufgeführt. Um darüber hinaus einen Eindruck von den positiven beziehungsweise negativen Speicherleistungen der Diatomeenzellen zu vermitteln, sind für die wichtigsten Ionen auch noch ihre Mediumkonzentration (nur in g-Ion/1) sowie das Verhältnis ihrer Konzentrationen innerhalb und außerhalb der Zellen (i : a) angegeben.

Der Summenquotient der im Zellsaft ermittelten Kationen- und Anionenkonzentrationen, der um ca. $11 \%$ über dem stöchiometrisch zu erwartenden Sollwert von 1 liegt, läßt ein Anionendefizit erkennen, das wahrscheinlich auf die Anwesenheit organischer Anionen sowie möglicherweise auch auf das Vorhandensein anionischer Nitrat- und Kieselsäurereste zurückzuführen ist, die durch die angewandten Nachweismethoden nicht erfaßt wurden.

Die osmotischen Potentiale des Zellsaftes $\left(\pi_{\mathrm{i}}\right.$ th.) und des Außenmediums ( $\pi_{\mathrm{e}}$ th.) wurden aus den Analysendaten unter Zugrundelegung eines osmotischen Aktivitätskoeffizienten von 0,9 errechnet. Ein Vergleich der aus diesen Werten berechneten Gefrierpunktserniedrigungen (GPE) mit den auf mikrokryoskopischem Wege bestimmten Werten ergibt für den Zellsaft ein Defizit von $-0,155^{\circ} \mathrm{C}=1,875 \mathrm{Atm}=7,5 \%$ 。 Dieser Fehlbetrag dürfte in erster Linie dem bereits erwähnten Vorhandensein der analytisch nicht erfaßten Anionen unbekannter Natur zuzuschreiben sein, die bei der Berechnung nicht berücksichtigt wurden. $\mathrm{Da}$ im übrigen für die Untersuchungen nur eine Probe zur Verfügung stand und damit die Möglichkeit einer Mittelwertbildung 
aus den Ergebnissen mehrerer Bestimmungen entfiel, dürfte der Fehlbetrag zu einem gewissen Teil zu Lasten der relativ großen Fehlerbreite gehen.

Der flammenphotometrisch bestimmte Li-Gehalt der Probe, dessen Umrechnung einen AFS-Wert von 50,2\% ergab, erschien zunächst unwahrscheinlich hoch. Zur Überprüfung der Verläßlichkeit dieses Wertes wurde deshalb das Volumen des interstitiellen Raumes von Glaskugeln, die in einen mit Wasser gefüllten Meßzylinder gegeben wurden, bestimmt. Dabei wurde ein Wert von $41,5 \%$ ermittelt, der beträchtlich von dem bei dichtester Kugelpackung zu erwartenden Wert $(10,7 \%$ ) abweicht. $\mathrm{Da}$ die Zellen des Untersuchungsmaterials, die alle etwa von gleicher Größe waren, annähernd zylindrische Form hatten, muß vermutet werden, daß der interstitielle Raum in diesem Falle noch größer war. Berücksichtigt man ferner noch, daß der als AFS bestimmte Raum ja auch noch den Imbibitionsraum der Zellwände und den frei lösenden Raum des Protoplasmas mitumfaßte, so erscheint der ermittelte Li-Wert durchaus annehmbar.

Demnach wäre nur die bei etwa 25 bis $30 \%$ der abgesiebten und mit der Li-Spüllösung behandelten Zellen beobachtete Reizplasmolyse als Unsicherheitsfaktor bei der Beurteilung der Analysenergebnisse in Betracht zu ziehen. Derartige Plasmolysen sind bei vielen Diatomeen vor allem durch mechanische und chemische Reize sehr leicht auszulösen (Gross 1940). Über den Mechanismus dieser Reaktion sowie über die sich dabei abspielenden Anderungen im Chemismus der Zelle ist jedoch so gut wie nichts bekannt. Falls die Plasmolyse nur zu einer durch Reizkontraktion des Protoplasmas bedingten Wasserabgabe führt, müßte die Zellsaftkonzentration im Verlauf dieses Vorganges ansteigen; bei gleichzeitiger Erhöhung der Permeabilität für die im Zellsaft gelösten Substanzen (vgl. auch STADELMANN 1956, p. 83 ff.) müßte die Konzentration jedoch gleichbleiben, da durch die während der Plasmolyse stattfindende Verkleinerung des Zellsaftraumes eine Endodiffusion von Stoffen aus dem umgebenden Medium zunächst verhindert wird. In beiden Fällen mïßten zumindest die Ionen$\mathrm{rel}$ a t i o $\mathrm{n}$ e $\mathrm{n}$ annähernd gleichbleiben.

Vergleicht man unter Berücksichtigung dieser erläuternden beziehungsweise einschränkenden Bemerkungen die in Tabelle 1 zusammengestellten Analysenergebnisse mit den an anderen Meeresalgen gewonnenen Resultaten, so muß man feststellen, daß der Zellsaft von Coscinodiscus wailesii keine außergewöhnlichen chemischen Qualitäten besitzt. Hier wie dort wird das Kalium als wichtigstes intrazelluläres Kation stark gespeichert, während Natrium im Zellsaft in bedeutend geringerer Konzentration vorliegt als im Außenmedium. Ammonium, das im Zellsaft von Noctiluca miliaris in relativ hoher Konzentration vorgefunden wurde (KESSELER 1966), und offenbar für den Auftrieb der Noctiluca-Zellen von Bedeutung ist, konnte bei $C$. wailesii überhaupt nicht nachgewiesen werden. $\mathrm{Ca}^{\prime}, \mathrm{Mg}^{\prime \prime}$ und $\mathrm{H}_{2} \mathrm{PO}_{4}{ }^{\prime}$, deren osmotische Konzentrationen insgesamt etwa $5 \%$ des mikrokryoskopisch bestimmten osmotischen Potentials des Zellsaftes ausmachen, waren ursprünglich sicher nicht in diesen Mengen darin enthalten, sondern wurden zum Teil wahrscheinlich erst nachträglich durch die Abtötungsund Präparationsmethoden aus anderen Zellbestandteilen freigesetzt.

Bei den Anionen ist lediglich der verhältnismäßig hohe Sulfatgehalt bei entsprechend reduzierter Chloridkonzentration bemerkenswert. Diese Verhältnisse erinnern in gewisser Weise an die bei Desmarestia viridis festgestellten Ionenrelationen. 
Auch der relativ niedrige $\mathrm{p}_{\mathrm{H}}$-Wert, der gemäß einer Bestimmung mit MERCK-Indikatorpapier bei $5 \mathrm{lag}$, legt einen solchen Vergleich nahe. Unter Berücksichtigung der puffernden Wirkung der $\mathrm{H}_{2} \mathrm{PO}_{4}$-Ionen und der starken Verdünnung der Saftprobe durch ein etwa gleiches Volum Li-Spüllösung darf man vermuten, daß der $\mathrm{pH}^{-}$Wert des nativen Saftes ca. 4 betragen haben mag.

Das spezifische Gewicht des Zellsaftes wurde an einer gemäß den Analysenergebnissen bereiteten künstlichen Salzlösung ermittelt. Es lag erwartungsgemäß über dem für das Kulturmedium bestimmten Wert. Setzt man unter Berücksichtigung der oben angedeuteten Fehlermöglichkeiten voraus, daß der native Zellsaft mit dem Außenmedium isotonisch war, so würden trotzdem allein schon die durch meine Untersuchungsmethoden ermittelten Ionen relationen bei Vergleich mit denen des Seewassers ein relativ größeres spezifisches Gewicht des Zellsaftes bedingen. Als Ursache zur Erklärung der vorwiegenden Verbreitung von $C$. wailesii in der euphotischen Zone kommt das spezifische Gewicht ihres Zellsaftes also nicht in Betracht. Gross \& ZEUTHEN (1948) hatten dies auf Grund eingehender physiologischer Untersuchungen an Ditylum brightwelli für ihr Objekt angenommen.

Eine Bestätigung dieser Annahme glauben auch BekLEMishev et al. (1961) durch ihre an nativen Zellsaftproben von Etbmodiscus rex ermittelten Analysenergebnisse gefunden zu haben. Die Zellen dieser Diatomee, einer nahen Verwandten der von mir untersuchten Art, bieten wegen ihrer für diese Organismengruppe geradezu gigantischen Größe ( $\varnothing$ bis zu $2 \mathrm{~mm}$ !) besondere Vorzüge für derartige Experimente. Mit Hilfe einer Ultramikrokolbenpipette konnten die Autoren je Zelle Saftmengen von 0,5 bis $1,5 \mathrm{~mm}^{3}$ gewinnen, wobei sich allerdings eine Vermischung mit dem wandständigen Zytoplasma nicht ganz vermeiden ließ. Die an diesen Saftproben gewonnenen Analysenergebnisse wurden mit Hilfe ultramikrochemischer Nachweismethoden (Petrikova 1959) erarbeitet.

Unter Berücksichtigung dieser günstigen Umstände wäre es nur zu verständlich, den Ergebnissen der russischen Forscher ein größeres Gewicht beizumessen als meinen auf indirektem Wege ermittelten Resultaten. Eine eingehende kritische Besprechung ihrer Methoden und Ergebnisse erscheint daher angebracht.

Zur Ermöglichung einer raschen Orientierung wurden die Befunde der russischen Autoren in die von mir bevorzugte Einheit Val/l umgerechnet und in Tabelle $2 \mathrm{zu}$ sammengestellt. Aus dieser Darstellung ist zunächst zu ersehen, daß das stöchiometrische Verhältnis der Kationen und Anionen sehr unterschiedlich ausfällt; während es bei den ersten beiden Zellen infolge des unwahrscheinlich niedrigen Chloridgehaltes, der im allgemeinen nur wenig von der Mediumkonzentration abweicht (vgl. Proben 3 bis 5), größer als 1 ist, liegt es bei den übrigen Proben wegen des enormen Kationendefizits im Mittel etwa bei 0,5. Mit Ausnahme von Zelle Nr. 4, die etwas aus dem allgemeinen Rahmen fällt, war der Gehalt der Proben an Natrium (bestimmt als Natrium-Uranylazetat) um ca. 30\% niedriger als im Außenmedium, ein Befund, der im Hinblick auf die zumeist negative $\mathrm{Na}$-Speicherung der meisten Meeresalgen vertretbar erscheint. Allerdings ist es unverständlich, wieso gerade die als "tot" bezeichnete Zelle Nr. 7 einen noch geringeren Na-Gehalt aufweist als die lebenden. Umgekehrt verhält es sich mit dem zellphysiologisch wichtigen Kalium [bestimmt als KaliumNatrium-Kobalt(III)-Nitrit], das von lebenden Zellen zumeist stark gespeichert wird. 


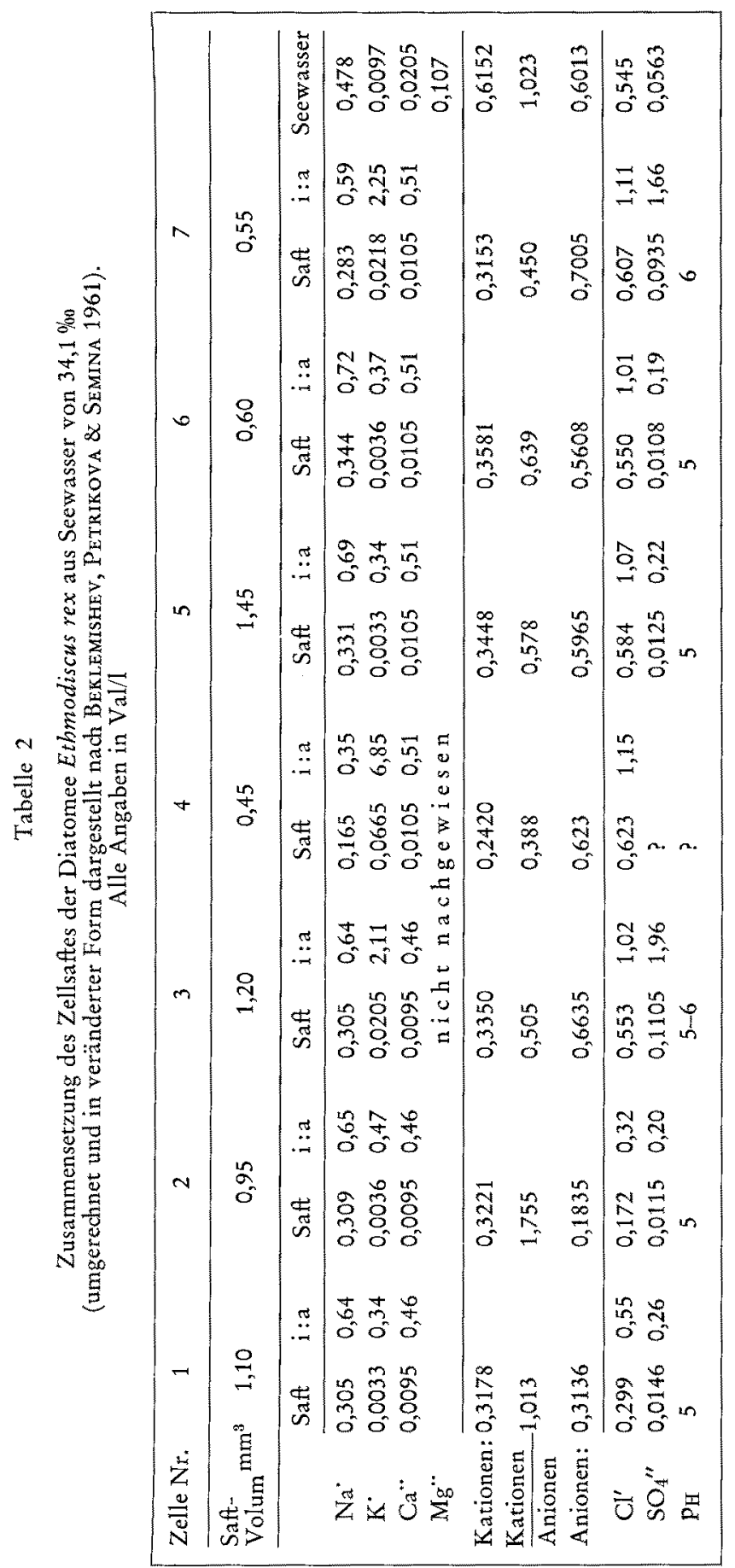


Für dieses Ion wurde - abgesehen wieder von Probe Nr. 4 - ausgerechnet in der toten Zelle Nr. 7 ein höherer Gehalt ermittelt.

Gute Ubereinstimmung zeigen allein die Ca"-Werte, die durch zwei verschiedene Analysenverfahren ermittelt wurden: einmal komplexometrisch mit ADTE (Äthylendiamintetraessigsäure) unter Verwendung von Murexid als Indikator, zum anderen nach Auflösung der Ca-Oxalatfällung durch konduktometrische Rücktitration der im Überschuß zugegebenen Ce(IV)-Ionen mit einer Lösung von Mohrschem Salz. Die Werte liegen allerdings beträchtlich niedriger als meine Angaben, was teilweise darauf zurïckzuführen sein mag, daß durch meine Methode der Saftgewinnung möglicherweise auch das im Protoplasma beziehungsweise in den Zellwänden gebundene Ca" miterfaßt wurde. $\mathrm{Mg}^{*}$ konnte von den russischen Forschern mit Hilfe der TitangelbReaktion nicht nachgewiesen werden. $\mathrm{Zu}$ erwähnen bleibt noch der Sulfat-Gehalt der Proben, der mit Hilfe einer besonderen, von Bertolacini \& BARNey (1967) ausgearbeiteten Methode durch Barium-Chloranilat kolorimetrisch bestimmt wurde. Bei den lebenden Zellen liegt er weit unter dem von mir ermittelten Wert. Für die geschädigte (Nr. 3) beziehungsweise abgestorbene Zelle (Nr. 7) hingegen wurde auch in diesem Falle eine höhere Konzentration gefunden als im Außenmedium.

Angesichts dieser großen Unterschiede der Analysenergebnisse erscheint es zur Zeit unmöglich, die noch weitaus beträchtlicheren Abweichungen von meinen Resultaten auf eine gemeinsame Ursache zurückzuführen. Da die russischen Autoren ausdrücklich darauf hinweisen, daß mit den von ihnen angewandten Nachweisverfahren bei Parallelbestimmungen an ein und derselben Saftprobe (!) wie auch an Seewasserproben Ergebnisse erzielt wurden, die mit den Erwartungswerten gut übereinstimmten, was bei der Kleinheit der zur Verfügung stehenden Probenvolumina erstaunlich ist, könnte allenfalls die Art und Weise der Vorbehandlung des Materials Hinweise auf mögliche Ursachen für die so unterschiedlichen Ergebnisse liefern. Die Zellen wurden nämlich vor der Saftextraktion mit Hilfe einer Pipette einzeln auf eine Glasplatte übertragen und dort mit Filtrierpapier abgetrocknet. Diese Behandlung könnte bei den gegenüber verschiedensten Reizen recht empfindlichen Diatomeen (Gross 1940) Spontanplasmolysen bewirkt haben, die zu einer Vermischung ihres Zellsaftes mit noch anhaftendem Seewasser führten. Allerdings wäre damit noch keine plausible Erklärung für das zum Teil stark gestörte stöchiometrische Verhältnis der Kationen und Anionen gefunden. Zur Beantwortung all dieser noch offenen Fragen bedarf es daher dringend weiterer Untersuchungen an Diatomeen, die am hiesigen Institut in Kürze wiederaufgenommen werden sollen.

\section{ZUSAMMENFASSUNG}

1. Die vorläufigen Ergebnisse von chemischen Analysen des Zellsaftes von Coscinodiscus wailesii werden mitgeteilt und den von BeKLEMishev et al. (1961) an Zellsaftproben von Ethmodiscus rex gewonnenen Resultaten gegenübergestellt.

2. Eine eingehende kritische Diskussion der beiderseitigen, völlig widersprüchlichen Ergebnisse liefert keine Anhaltspunkte zur befriedigenden Erklärung dieser Diskrepanzen, die weiteren Untersuchungen vorbehalten bleiben muß. 
Meinem ehemaligen Mitarbeiter, Herrn J. K. HotrmanN, danke ich für die gewissenhafte Ausführung der chemischen Analysen. Herrn Dipl.-Bibliothekar W. MeIss bin ich für seine Bemühungen um die Ubersetzung des russischen Textes zu Dank verpflichtet.

\section{ZITIERTE LITERATUR}

BERTolacinI, R. J. \& BARNEY, J. E., 1957. Colorimetric determination of sulfate with barium chloranilate. Analyt. Chem. 29, 281-283.

Beklemishev, C. W., Petrikova, M. N. \& Semina, H. J., 1961. On the cause of the buoyancy of plankton diatoms. Trudy Inst. Okeanol. 51, 33-36.

Gross, F, 1940. The osmotic relations of the plankton diatom Ditylum brigbtwelli (West.). J. mar. biol. Ass. U. K. 24, 381-415.

- \& ZEUTHEN, E., 1948: The buoyancy of plankton diatoms: a problem of cell physiology. Proc, R. Soc. (Ser, B) 135, 382-389.

Kessteler, H., 1958. Eine mikrokryoskopische Methode zur Bestimmung des Turgors von Meeresalgen. Kieler Meeresforsch, 14, 23-41.

- 1964. Zellsaftgewinnung, AFS (apparent free space) und Vakuolenkonzentration der osmotisch wichtigsten mineralischen Bestandteile einiger Helgoländer Meeresalgen. Helgoländer wiss. Meeresunters. 11, 258-269.

- 1965. Turgor, osmotisches Potential und ionale Zusammensetzung des Zellsaftes einiger Meeresalgen verschiedener Verbreitungsgebiete. (Proc. 5th Mar. Biol. Symp.) Botanica Gotboburg. 3, $103-111$.

- 1966. Beitrag zur Kenntnis der chemischen und physikalischen Eigenschaften des Zellsaftes von Noctiluca miliaris. Veröff. Inst. Meeresforsch. Bremerb. (Sonderbd.) 2, 357-368.

LEWIN, J. C., 1965. The boron requirement of a marine diatom. Naturwissenschaften 52, 70-71.

Petrikova, M. N., 1959. Ultramicrochemical study of the composition of the cell sap of plankton diatoms. Z. analit. Chim. 14, 255-257.

Smayda, T. H. \& Boleyn, B. J., 1965. Experimental observations on the flotation of marine diatoms. I. Limnol. Oceanogr. 10, 499-509.

Stadelmann, E., 1956. Plasmolyse und Deplasmolyse. In: Handbuch der Pflanzenphysiologie. Hrsg. von W. Ruhland. Springer, Berlin 2, 71-115. 